\title{
Partially thrombosed multiple pulmonary artery aneurysm secondary to Behçet disease
}

\author{
Ibrahim Guler MD, Kemal Odev MD, Recep Tunc MD
}

\begin{abstract}
$\mathrm{A}^{23}$
23-year-old man with an established diagnosis of Behçet disease

(BD) for five years presented with cough and hemoptysis. The laboratory findings were normal with the exception of mild leukocytosis. An anteroposterior radiograph revealed multiple round lesions (Figure 1). He underwent multidetector computed tomography (MDCT) to determine the cause of hemoptysis. MDCT demonstrated multiple pulmonary artery aneurysms (PAAs) bilaterally (Figures 2A and $2 \mathrm{~B}$ ). Alveolar hemorrhage (Figure $3 \mathrm{~A}$ ) and subpleural fibroticatelectatic changes (Figure $3 \mathrm{~B}$ ) were also detected within the pulmonary parenchyma on images reconstructed using a lung algorithm.

$\mathrm{BD}$ is a multisystem disorder first described by Hulusi Behçet in 1937. It is a vasculitis that presents with a triad of findings including recurrent ulcers of the oral and genital mucosa with relapsing uveitis. Additional clinical manifestations were later described and include involvement of the skin, joints, large vessels, lung, brain, and gastrointestinal and genitourinary tracts (1). The underlying pathological process in $\mathrm{BD}$ is perivascular inflammation affecting vessels of different sizes in various organs. The inflammatory process is acute and results in destruction of the walls of the affected vessels. BD can cause aneurysms that can rupture (1). The most commonly affected arteries are the aorta, the pulmonary arteries and the femoral artery.

Vascular manifestations of $\mathrm{BD}$ in the thorax are observed in both the arterial and venous system. Venous involvement is, in fact, observed more frequently than arterial involvement. Occlusion with or without evidence of thrombosis of the superior vena cava or brachiocephalic vein are quite common and, less frequently, occlusion and/or stenosis
\end{abstract}

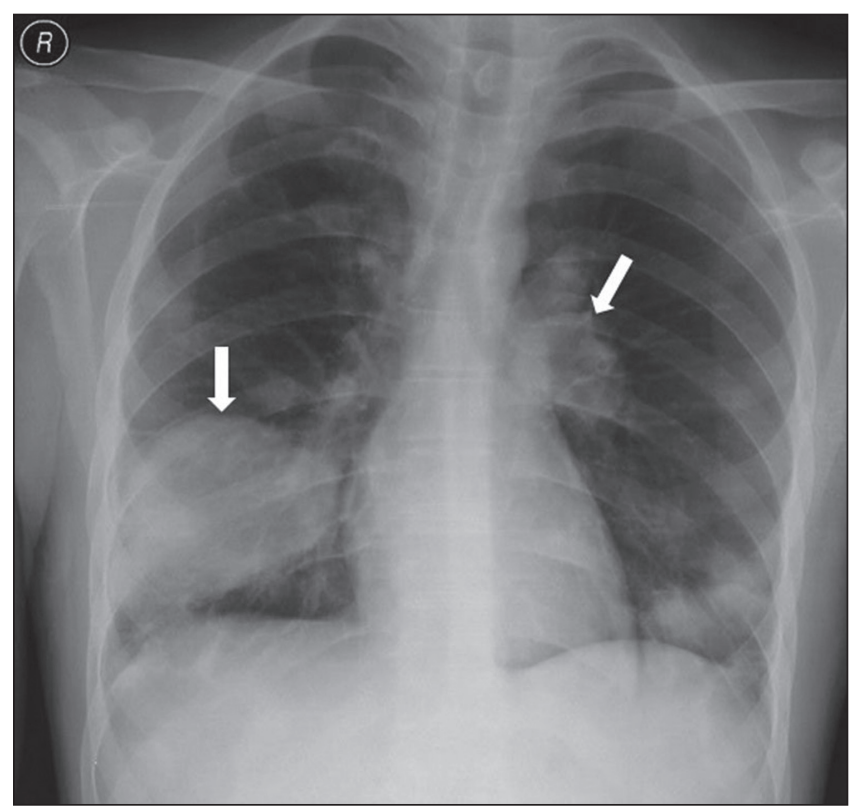

Figure 1) Anteroposterior chest radiograph showing multiple wellcircumscribed nodular lesions (arrows)

and aneurysms of the pulmonary arteries can occur. PAA is the most common lung manifestation of $\mathrm{BD}$, which is the most common cause of PAAs. Additional parencymal findings, including atelectasis, hemorrhage and infarction (focal or subpleural consolidation), have also been reported. These are commonly encountered secondary to thrombosis of the pulmonary vessels. Pneumonia, bronchitis, fibrosis, subpleural infiltrates, subpleural nodules, pleural effusion and emphysema have been described $(2,3)$.

Clinically, patients most frequently present with hemoptysis secondary to PAA. This is more commonly observed in male patients with BD.

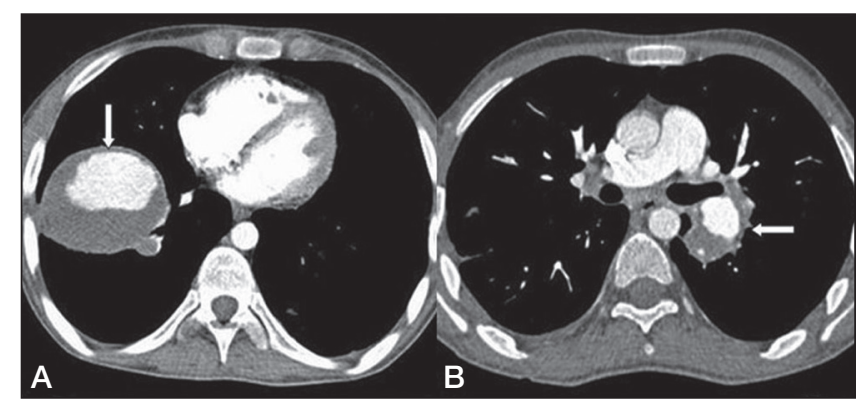

Figure 2) Axial contrast-enhanced computed tomography scan showing partial thrombosed pulmonary arteries in the right (A) and left (B) lung (arrows)

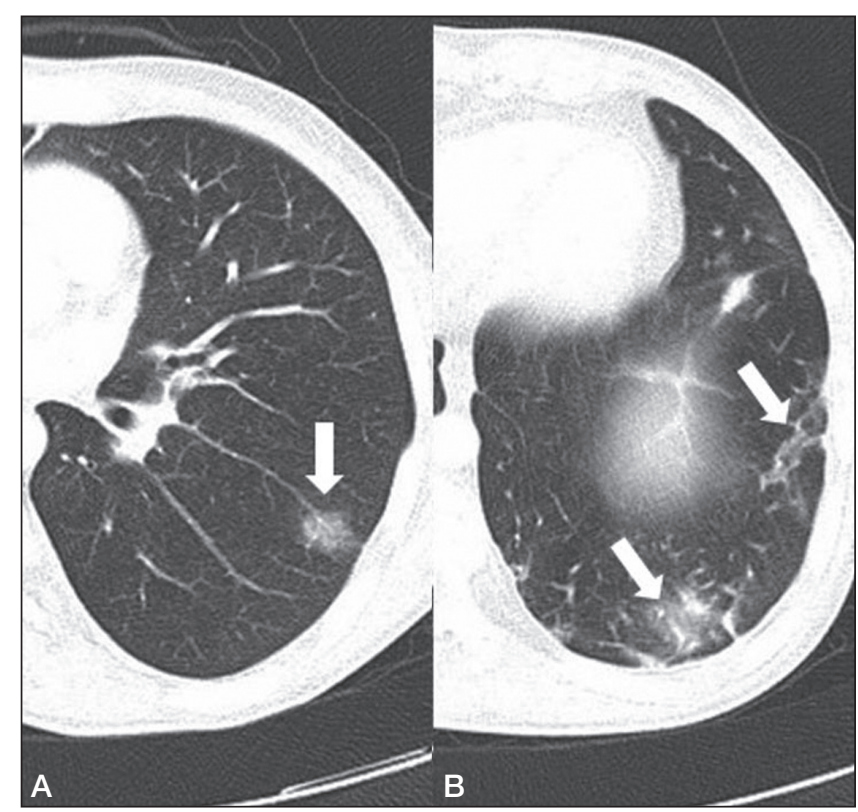

Figure 3) Alveolar hemorrhage (A) and subpleural fibrotic-atelectatic changes (B) are also detected on parencyma in lung window (arrows)

Selcuk University Faculty of Medicine, Selcuklu, Konya, Turkey

Correspondence: Dr Ibrahim Guler, Selcuk University Faculty of Medicine, Selcuklu, Konya, Turkey 42075.

Telephone 90-332-224-3800,fax 90-332-224-3800,e-mail dribrahimguler@gmail.com 


\section{KEY LEARNING POINTS}

- $\mathrm{BD}$ is a multisystem and chronic inflammatory disorder of unknown etiology.

- The main pathological process is vasculitis and perivascular inflammatory infiltrates affecting the blood vessels.

- Vascular system involvement is observed in $20 \%$ to $40 \%$ of patients. Venous and arterial involvement are seen in BD, with venous involvement more common.

- PAA is the most frequent form of thoracic involvement, with hemoptysis being the most common clinical presentation

\section{REFERENCES}

1. Erkan F, Gul A, Tasali E. Pulmonary manifestations of Behcet's disease. Thorax 2001;56:572-8.

2. Hiller N, Lieberman S, Chajek-Shaul T, Bar-Ziv J, Shaham D. Thoracic manifestations of Behçet disease at CT. RadioGraphics 2004;24:801-8.

3. Ceylan N, Bayraktaroglu S, Erturk SM, Savas R, Alper H. Pulmonary and vascular manifestations of Behcet disease: Imaging findings. AJR Am J Roentgenol 2010;194:158-64.

The 'Images in Respiratory Medicine' section of the Canadian Respiratory Journal aims to highlight the importance of visual interpretation, whether physiological, radiological, bronchoscopic, surgical/thorascopic or histological, in the diagnosis of chest diseases. Submissions should exemplify a classic, particularly dramatic or intriguing presentation of a disease while offering an important educational message to the reader (insightful diagnostic pearls or differential diagnosis, etc). This section is not intended to be a vehicle for publication of case reports (see the Clinical-Pathologic-Conferences for case-based leaning series). 


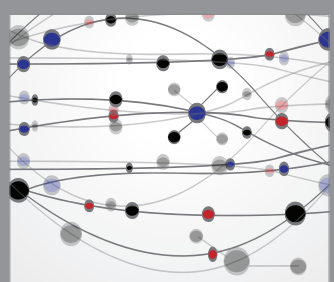

The Scientific World Journal
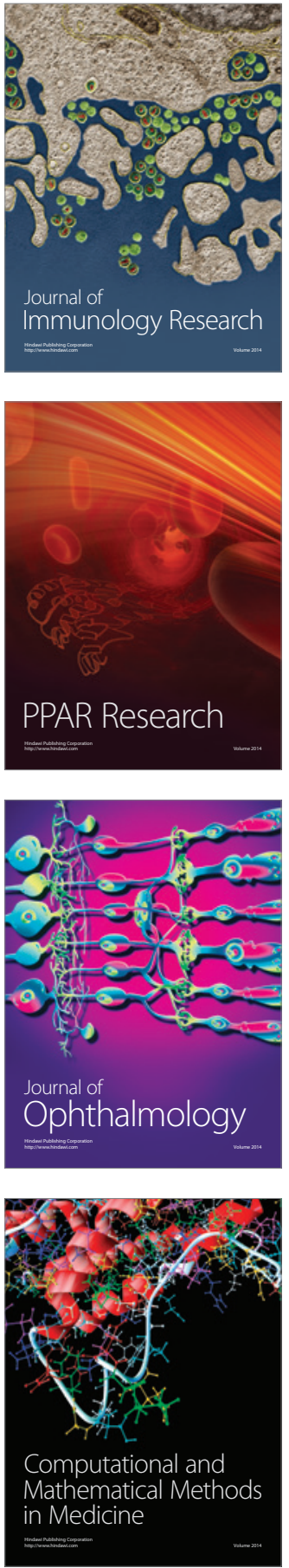

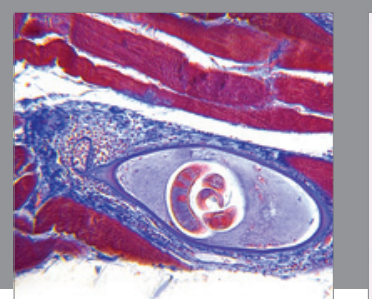

Gastroenterology Research and Practice

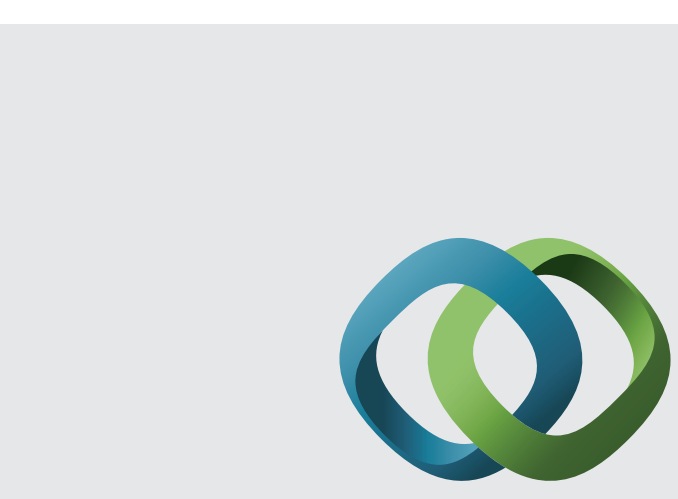

\section{Hindawi}

Submit your manuscripts at

http://www.hindawi.com
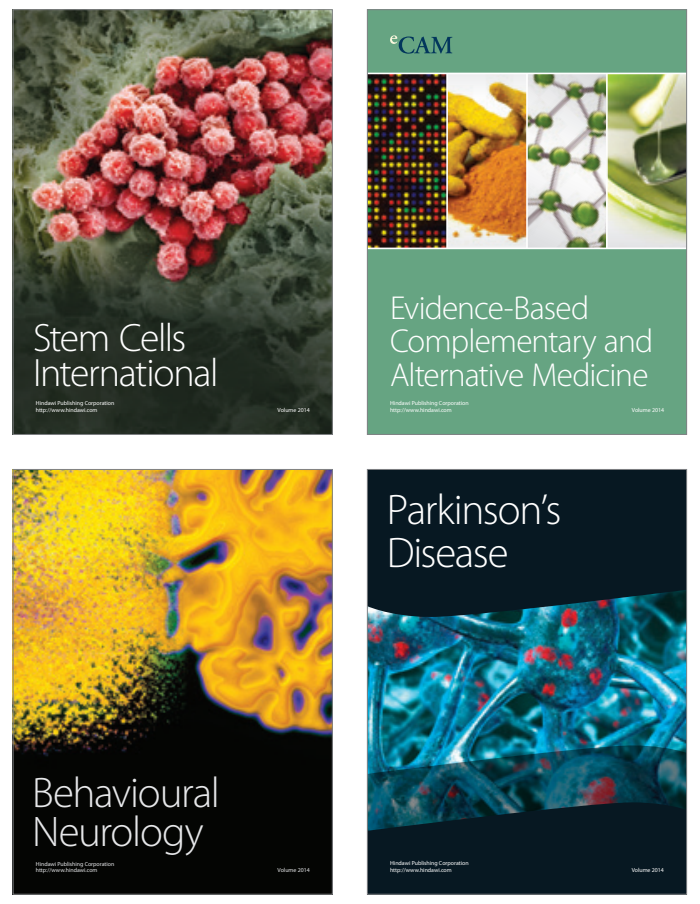
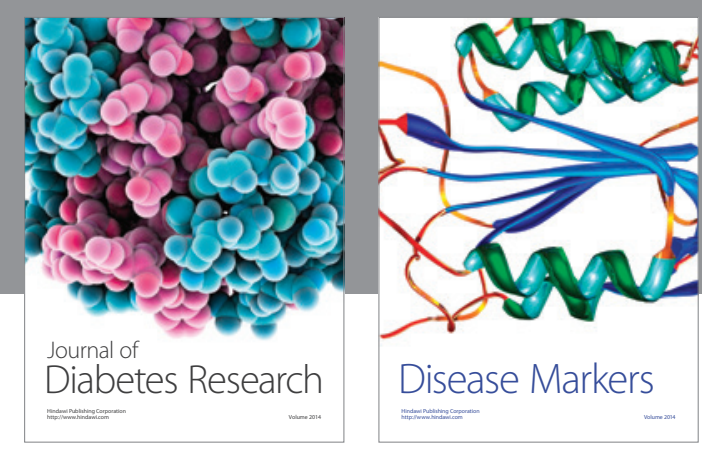

Disease Markers
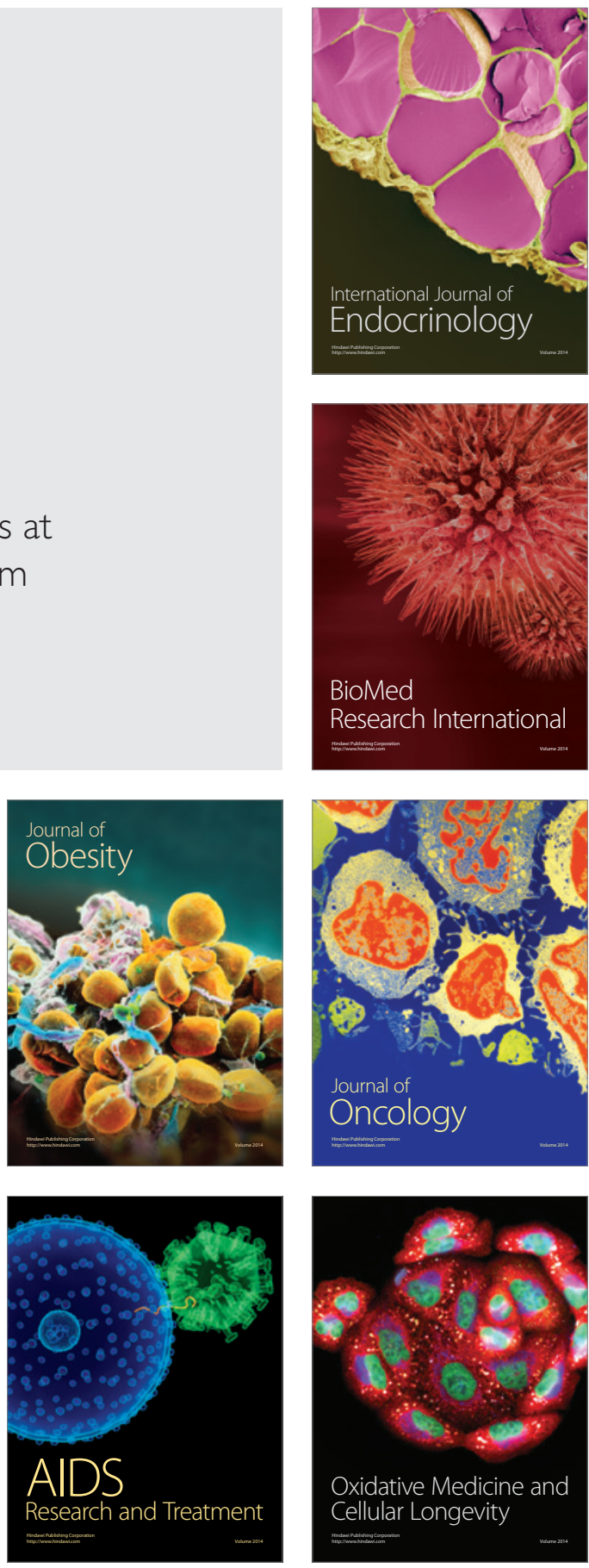